\title{
IN SOMNIA IN THE ELDERLY: EVALUATION AND MANAGEMENT
}

\author{
Dr Matthew Ng Joo Ming, Dr Ng Beng Yeong
}

\section{ABSTRACT}

Sleep disturbance is common in the elderly and is frequently undiagnosed. It has been estimated that 75 percent of adults $>65$ years of age has sleep disturbance and 30 percent of them has insomnia. The classification of insomnia has less significance in the older adults as the subtypes demonstrate significant overlap and usually treatment of the underlying disorder does not solve the problem or cure it. The elderly has multiple comorbidities and poly pharmacy with a myriad of cause for insomnia. A comprehensive medical and psychiatric history together with a complete physical examination and mental state examination should be done in the evaluation of the older patient. Behavioural therapy with sleep hygiene education should be the initial treatment together with the treatment of the contributing physical and psychiatric conditions. Referral to an expert for cognitive behavioural therapy or multicomponent therapy may be necessary if the initial therapy failed to produce any improvement. If medications are needed it can be combined with behavioural therapy. Medication used should be the lowest effective dose and prescribed for shortterm use of not more than four weeks. Medications used need to be discontinued gradually and one needs to be mindful of rebound insomnia upon withdrawal. W henever possible, it will be ideal to avoid benzodiazepines and other sedative hypnotics as first choice for insomnia. 0 ver the counter sleep aids which usually contain antihistamines may not be good choices as they carry significant risk of adverse events and drug interactions. Currently the safest medications for use in the elderly includes the Z-drugs (zolpidem, zopiclone), melatonin and low dose tricyclic antidepressant Doxepin.

\section{Keywords: Insomnia, elderly, behavioural therapy, education}

\section{INTRODUCTION}

In an observational study published in 2016 by Tan et $\mathrm{al}^{1}$ on 350 individuals aged between 21 to 80 surveyed at SingHealth Polyclinics, 44 percent of them have inadequate sleep on weekdays and 26 percent of them have inadequate sleep on weekends. In fact, Singaporeans are the third most sleep deprived country after Japan and Korea. The amount of sleep varies between the age group, and adults need about seven to eight

\footnotetext{
Matthew Ng Joo Ming

Head and Senior Consultant

Family Medicine Continuing Care, Singapore General Hospital
}

\section{Ng Beng Yeong}

Consultant Psychiatrist

Ng Beng Yeong Psych Medicine Clinic

Mount Elizabeth Medical Centre hours. The prevalence of insomnia in the elderly (that is persons older than 65) has been estimated to be about 30 percent; the high prevalence is not surprising given the physiological related changes such as reduction of sleep efficiency and comorbidities associated with insomnia. FK Chui et $\mathrm{al}^{2}$ surveyed 1034 elderly in Hong Kong and found that occasional or persistent sleep disturbance were reported by 75 percent and insomnia in 38.2 percent of elderly. Advancing age is associated with a higher rate of sleep disturbance while females had a high rate of insomnia. Women are twice likely than men to have insomnia and prevalence increases with hormonal changes such as pregnancy and menopause. Patients with comorbidities such as pulmonary disease, heart failure, and painful condition are at increased risk. Sleep disturbance in elderly are often undiagnosed and inadequately treated. ${ }^{3}$ It has a significant impact on the quality of life; it leads to fatigue, cognitive impairment, mood disturbance, daytime sleepiness, behavioural problems, decreased motivation, increased errors of judgement and concerns about sleep. All these can lead to increased rate of accidental injuries and falls.

\section{DEFIN ITION}

Insomnia is defined as inability to fall asleep, the inability to stay asleep, or waking up earlier than desired. In order to make a correct diagnosis of insomnia the patient must have adequate opportunity to sleep and has an adequate and conducive place to sleep. Insomnia can be classified on the basis of the duration, acute (<four weeks) vs. chronic, severity (mild or severe), sleep components affected (impaired sleep onset, sleep maintenance or both), and cause (situational related to stress), primary or secondary to other medical or psychiatric disorders. However, these subtypes demonstrate considerable overlap and differentiation is no longer important in the elderly as treatment of the medical conditions that precipitate the insomnia may not necessarily provide a cure as there are perpetuating factors that need to be addressed as well. Most diagnosis can be made through careful history and focused physical examinations. Testing such as polysomnography and survey tools should be reserved for those whose history suggest a particular medical condition such as obstructive sleep apnoea syndrome, restless leg syndrome or narcolepsy etc. Treatment can be targeted at the underlying condition if any, in addition to applicable techniques involving sleep hygiene education, nonpharmacologic interventions and drug treatment if needed. It must be stressed that secondary insomnia can coexist with the primary sleep disorders and they must be addressed to optimise the treatment efforts. The two main diagnostic criteria used for the diagnosis of insomnia are the International Classification of Sleep Disorders (ICSD-3) and the Diagnostic and Statistical Manual of Mental Disorders (DMS 5) (Table 1). 


\section{Table 1: Diagnostic Criteria for Insomnia}

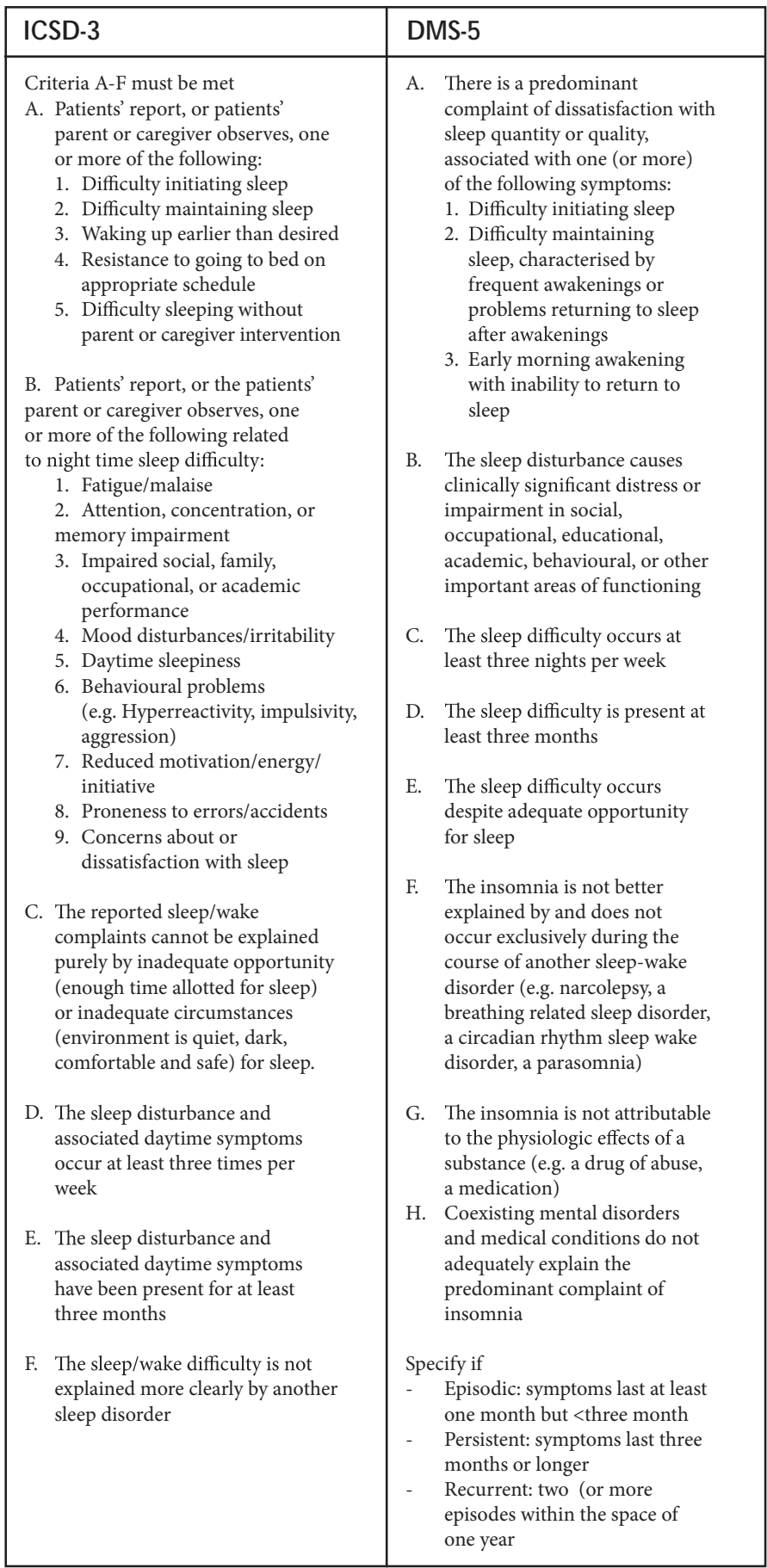

\section{RISK FACTORS FOR IN SO MN IA}

Insomnia can occur alone or as a symptom complex associated with a comorbid condition. Multiple factors increase the risk for insomnia in the older adults. They include environmental, behavioural, medical, and social factors (Table 2). As people age, they tend to have multiple co-morbidities and will be on multiple medications from different specialists that increases their risk of developing insomnia. As the older adults retire, they may change their usual bedtime and wake time to suit their new lifestyle, putting them at risk of developing insomnia. Patients with movement disorders (e.g. restless leg syndrome, periodic limb movement disorder), circadian rhythm disorders or breathing disorders (e.g. obstructive sleep apnoea) may present with insomnia ${ }^{4}$. Therefore, these conditions need to be identified and treated. Treatment of these conditions alone may not always resolve the insomnia. Pulmonary disease, chronic pain, heart failure, gastrointestinal reflux and prostate problems are common in the elderly and often times associated with insomnia. Illicit drug use, cigarette smoking, alcohol consumption can also cause insomnia. Insomnia is a strong predictor for the development of depression, anxiety and drug or alcohol abuse. It is also highly predictive of relapse of these condition. Depression usually improves more rapidly in a patient with insomnia if both conditions are treated concomitantly..$^{5,6}$

\section{Case illustration:}

A 69-year-old Chinese lady lives alone with a Filipino helper. She is independent in her daily activities of living. She has been on follow-up at the family medicine clinic for the past five years for the following medical problems: A trial fibrillation, ischemic heart disease, hyperlipidaemia and hypertension. She has a history of nasopharyngeal carcinoma treated by radiation therapy in 1994, thyroidectomy for multi nodular goitre in 1993 and laparoscopic cystectomy for ovarian cysts. She also has Syndrome of Inappropriate Antidiuretic Hormone (SIADH) diagnosed in 2014 and is put on fluid restriction. In December 2018 she was admitted to the restructured hospital for right ankle swelling and redness which was diagnosed as cellulitis and treated with a course of clindamycin. Incidentally during admission, she was found to have persistently low serum sodium level despite fluid restriction for SIADH. A synacthen test administered diagnosed adrenal insufficiency and she is started on hydro cortisone replacement. She is currently on the following medications: warfarin $2.5 \mathrm{mg}$ om; Levothyroxine $50 \mathrm{mcg}$ om; simvastatin $10 \mathrm{mg}$ on; bisoprolol $1.25 \mathrm{mg}$ om; omeprazole $20 \mathrm{mg}$ om and hydrocortisone $10 \mathrm{mg}$ om and hydrocortisone $5 \mathrm{mg}$ at 4 pm.

Upon discharge from the ward, she was reviewed by the endocrinologist at the specialist outpatient clinic and continued on the same dosage of hydrocortisone and thyroxine. She returned to the family medicine clinic three months (March 2019) after discharge for review and for topping up of the rest of her medication. During consultation, she complained that she had not been able to sleep for the past three months and instinctively she stopped one of her medications and she had been able to sleep blissfully. This put the clinician at a dilemma as the medication is essential to treat her underlying medical condition.

\section{Question:}

Which medication did she stop? 


\section{Table 2: Comorbidities, Conditions, and Substances associated with Insomnia}

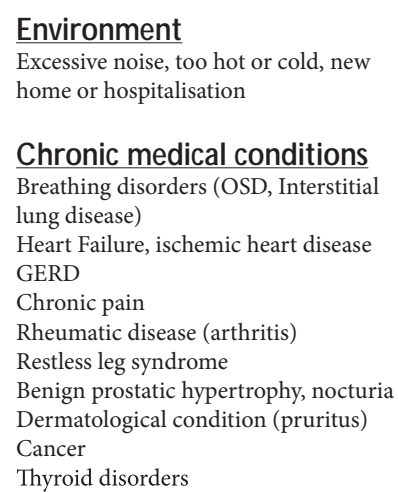

Excessive noise, too hot or cold, new

home or hospitalisation

Chronic medical conditions

Breathing disorders (OSD, Interstitial lung disease)

Heart Failure, ischemic heart disease GERD

Chronic pain

Rheumatic disease (arthritis)

Restless leg syndrome

Benign prostatic hypertrophy, nocturia

Dermatological condition (pruritus)

Cancer

Thyroid disorders

\section{Medications}

Antidepressants, antihypertensives

Appetite suppressants

Beta agonist

Caffeine

Diuretics

Glucocorticoids

Theophylline, salbutamol

Sedative and hypnotics

Antihistamines, cough medications

\section{Psychiatric conditions}

Anxiety, depression

Post-traumatic stress disorder

\section{Substance abuse}

Alcohol, Illicit drugs, tobacco

\section{EVALUATION}

Insomnia is a clinical diagnosis. In addition to the usual medical and psychiatric history, a detailed sleep history should also be obtained from the patient, partner or carer. The sleep history should include details such as the timing of the insomnia, the daytime effects and symptoms, the sleep schedule, the sleep environment and the patient's sleeping habits. The 3 P's of insomnia: predisposing, precipitating and perpetuating factors should be included in the history taking (Table 3). Relevant personal and social factors such as isolation, loneliness, bereavement, change in residence and financial concerns, are key factors in the history and may indicate a temporary situational insomnia. Further, a complete physical examination, including neurological and mental state assessment should be conducted to look for possible underlying medical conditions e.g. a raised jugular venous pressure (JVP) and lung crackles and oedema of heart failure. Referral to a psychogeriatrician and other specialist may be needed when the history and physical suggest sleep apnoea, restless leg syndrome, narcolepsy and REM sleep behaviour disorder.

\section{Case illustration}

In the patient above she correctly diagnosed herself that her inability to sleep properly for the past three months was due to her medications. The only new medications that had been started for her was hydrocortisone for adrenal insufficiency. The two medications in her drug regime that can disrupt sleep are thyroxine and hydrocortisone. However, she had been taking thyroxine replacement for a number of years and there had been no prior problem with it. She stopped the hydrocortisone on her own and slept blissfully. However, this created a therapeutic dilemma as the hydrocortisone is for treatment of her adrenal insufficiency. Not having enough glucocorticoids in the body will predispose her to electrolytes abnormalities, hypotension, and inability to handle stress and mount an effective response during an infection.

Not all patient diagnosis is as straightforward as the patient in the case illustration. In the elderly, insomnia can occur alone or as a symptom complex associated with multiple comorbidities. In such situation, keeping a two-week sleep diary (Table 4) provides information regarding patient's activities, bedtime routine, sleep quality, daytime symptoms and the use of causative substances. The information can confirm insomnia and allow the physician to provide specific behavioural guidance. The ultimate treatment goal is to improve sleep qualitatively and quantitatively, decrease related stress, and improve functioning in the day.

\section{Table 3: 3P's of insomnia}

\begin{tabular}{|c|c|}
\hline $\begin{array}{l}\text { Predisposing factors } \\
\text { - Age } \\
\text { - } \text { Easy arousability } \\
\text { - } \text { Female } \\
\text { - Living alone } \\
\text { - Psychological disorders: anxiety, } \\
\text { depression, substances abuse } \\
\text { - Smoking } \\
\text { Perpetuating factors } \\
\text { - } \text { Behavioural issues: excessive } \\
\text { time spent in bed, napping, } \\
\text { chronic medication use } \\
\text { - Psychological issues: worry } \\
\text { about sleep loss }\end{array}$ & $\begin{array}{l}\text { Precipitating factors } \\
\text { - } \text { Alcoholism } \\
\text { - Chronic pain } \\
\text { pulmorbid condition: Diabetes, } \\
\text { heart disease, thyroid disease, } \\
\text { heart failure } \\
\text { - Divorce, separation, loss of } \\
\text { spouse } \\
\text { - Lower social economic status } \\
\text { - Shift work } \\
\text { - Stroring } \\
\text { - Substance abuse } \\
\text { - Unemployment }\end{array}$ \\
\hline
\end{tabular}

\section{Table 4: Sleep diary}

\begin{tabular}{|c|c|c|c|c|c|c|c|}
\hline Activity/day & Monday & Tuesday & W ednesday & Thursday & Friday & Saturday & Sunday \\
\hline \multicolumn{8}{|l|}{ Daytime activity and Pre-sleep ritual } \\
\hline \multicolumn{8}{|l|}{ Naps (Number) } \\
\hline \multicolumn{8}{|l|}{ Exercise (type/duration) } \\
\hline \multicolumn{8}{|l|}{ Alcohol/caffeine (amount) } \\
\hline \multicolumn{8}{|l|}{ Food and drink } \\
\hline Stress irritability before bedtime & none & some & moderate & high & & & \\
\hline \multicolumn{8}{|l|}{ Medications/sleep aids } \\
\hline \multicolumn{8}{|l|}{ Activities hour before bedtime } \\
\hline \multicolumn{8}{|l|}{ Bed time (lights out) } \\
\hline \multicolumn{8}{|l|}{ Sleeping and getting back to sleep } \\
\hline \multicolumn{8}{|l|}{ Wake up time } \\
\hline \multicolumn{8}{|l|}{ Sleep breaks } \\
\hline Quality of sleep & $0=$ worst & $10=$ best ever & & & & & \\
\hline Total sleep time & & & & & & & \\
\hline
\end{tabular}




\section{MANAGEMENT}

The goal of treatment of the insomnia is the improvement of sleep quality and or quantity, and the reduction in insomnia related daytime impairments. The treatment should be a shared decision between the physician and patient. They should be involved in the formulation of the plan about the treatment goals and choice of treatment. The therapy will depend on the patient's willingness to engage in the behaviour therapies and the possibility of side effects from the medications use. Therefore, co-operation from patient is crucial for success. In the elderly, behavioural interventions are effective and recommended as an initial treatment of insomnia. Behavioural therapy and non-pharmacologic interventions appear to have better longterm efficacy with fewer side effects than pharmacologic interventions. Short term medical therapy is considered when non-pharmacologic interventions are not effective. It is best not to use or select benzodiazepines or other sedative hypnotics as a first choice in the management of insomnia in older adults. Hypnotics have benefits of improving sleep quality, total sleep time, and frequency of night time awakening. However these short-term gains are small compared to the risk of cognitive and psycho motor adverse events.

\section{N on-pharmacological Treatment}

Cognitive-behavioural therapy for insomnia (CBT-I) is a multi-component-based intervention involving cognitive and behavioural techniques like stimulus control therapy, sleep restriction therapy, relaxation training, cognitive restructuring, and sleep hygiene education. CBT-I improves sleep based on constant, good quality patient orientated evidence. ${ }^{7}$ Most of these behavioural interventions overlap and has certain similarities (Table 5). The goal is to change patient's misconceptions, beliefs, and attitude that hinder sleep. CBT-I involves several sessions and can be delivered in various modes, in person face to face, in groups, through an app, via telephone or through self-help videos. Most of the behavioural interventions can be used in the family physician office but this is not commonly done as many lack the training and expertise. It is also time consuming and lack financial reimbursement.

\section{A. Sleep Hygiene Education}

Education is key in all virtually sleep disorders and it can be easily performed by the family physician in the consultation room. However, time needs to be invested as some of the behavioural interventions might be difficult for patients to accept or understand without careful explanation. Sleep hygiene education is recommended as an initial intervention for all adults with insomnia so that personal habits and environmental factors that negatively impact sleep can be identified and corrected. ${ }^{8}$ Patients are advised to exercise regularly (but not within four hours of bedtime); avoid eating large meals and limit intake of fluid in the evening: avoid caffeinated drinks, tobacco, and alcohol intake four to six hours before bedtime. The bedroom should be used for sleep and sex only. One needs to maintain a regular sleep wake routine and avoid day time naps. The room should be comfortable with a cool temperature and there should not be noises that can disrupt the sleep (Table 6).

\section{B. Stimulus Control Therapy}

Stimulus control helps to establish a regular sleep-wake cycle and associate the bedroom with sleep. Specific instructions given to patients include the following: to lie down to sleep only if you feel sleepy; use the bed for sleeping and sex only; do not watch TV in bed or eat in bed; leave the bed if unable to fall asleep within 20 minutes and return to bed only when sleepy. It is also essential to establish a regular sleep-wake routine by setting the alarm at the same time every morning regardless of how many hours of sleep occurs. ${ }^{9,10}$

\section{Sleep Restriction Therapy}

This method is beneficial for patients who spent lots of time in bed trying to fall asleep. The idea is to limit the amount of time spent in bed to only the number of hours that the patient spends sleeping only. This time should not be less than five hours and it is gradually increased as sleep efficiency improves. This method has a risk of causing sleep deprivation and excessive daytime sleepiness.

\section{Cognitive Restructuring Therapy}

This aims to reduce the worrying and change misconceptions associated with sleep and insomnia using Socratic questioning. It changes how the older adult perceive the effect of sleep on their lives by challenging inaccurate patterns of thinking. The patient also uses a journal to record down troubling thoughts and worries before bedtime, the transfer of thoughts to paper may help the patient clear his or her mind making it easier to fall asleep.

\section{E. Relaxation Therapy}

The relaxation techniques used include deep breathing exercises, progressive muscle relaxation, biofeedback, and guided imagery. This aims to reduce tension and reduce intrusive thoughts that interfere with the ability to fall asleep. Mindfulness-based stress reduction techniques is a form of relaxation therapy aim to change reactions to stress by teaching purposeful awareness and acceptance of the present state. It includes breathing, body scan, meditations, and yoga. ${ }^{11,12}$

Life style and complementary approaches have some benefits. Regular moderate intensity exercise improves the quality of sleep in older adults. A randomised controlled trial showed that low impact exercise like Tai-Chi reduces daytime sleepiness and improve sleep quality in older adults with moderate sleep problems. ${ }^{13}$ Bright light therapy may be used to restore the normal circadian rhythm by providing time-exposure to bright light source, which helps to delay sleep. Family Physician can recommend white light sources with a bluish tint during the time exposure during the time interval patients tend to be more tired. ${ }^{14}$ A study demonstrated that just two four-hour evening sessions of exposure to bright light can improve early morning awakenings for up to one month following the treatment. ${ }^{15}$ Acupuncture at specific points to stimulate the body has been shown to improve insomnia symptoms in older adults. ${ }^{16}$ 
Table 5: O verview of Behavioural Therapies for Insomnia

\begin{tabular}{|c|c|c|}
\hline $\begin{array}{l}\text { Sleep H ygiene } \\
\\
\text { - Exercise regularly but not four hours before } \\
\text { bedtime } \\
\text { - No large meals, limit fluid intake in evening } \\
\text { - No caffeinated and alcoholic drinks } \\
\text { - Maintaing regular sleep-wake cycle } \\
\text { - Avoid distracting stimuli at bedtime }\end{array}$ & $\begin{array}{l}\text { Stimulus Control } \\
\text { - Lie down to sleep only if sleepy } \\
\text { - Use bedroom for sleep and sex only } \\
\text { - Lvoid watching TV, use of handphone } \\
\text { - Maintain a consiste to sleep within } 20 \text { minutes } \\
\text { an alarm }\end{array}$ & $\begin{array}{l}\text { Sleep Restriction } \\
\text { - Limit time in bed to the time actually spent } \\
\text { sleeping, gradually increase as sleep efficiency } \\
\text { improves }\end{array}$ \\
\hline $\begin{array}{l}\text { Paradoxical Intention } \\
\text { - Advise to remain awake to alleviate the anxiety } \\
\text { associated with the pressure to sleep }\end{array}$ & $\begin{array}{l}\text { Relaxation Training } \\
\text { - Autogenic training: imagine a calm environment } \\
\text { - Imagery training: focus on pleasant image } \\
\text { - Hypnosis, meditation, yoga, breathing exercise, } \\
\text { progressive muscle relaxation } \\
\text { - Visual or auditory feedback }\end{array}$ & $\begin{array}{l}\text { Cognitive therapy } \\
\text { - Counselling } \\
\text { - Identify and replace dysfunctional belief } \\
\text { regrading sleep } \\
\text { - Use journal to write down thoughts }\end{array}$ \\
\hline $\begin{array}{l}\text { Cognitive behaviour therapy for insomnia } \\
\text { (CBT-I) and multicomponent therapy } \\
\text { - Combination of stimulus control, relaxation } \\
\text { therapy, sleep hygiene education and cognitive } \\
\text { therapy }\end{array}$ & & \\
\hline
\end{tabular}

Table 6: Sleep H ygiene Education: Instructions for patients

\begin{tabular}{|l|l|}
\hline Behavioural Patterns & Sleep Environment \\
\hline - Keep a regular sleep-wake schedule & . Identify snoring or disruptive bed partners \\
- De not go to bed unless sleepy & Keep bedroom cool and dark \\
- Exercise regularly (But not four hours before sleep) & Eliminate as much noise as possible in the sleeping room \\
- Increase exposure to natural light and bright light during the day and early & Place clocks out of sight \\
at night & To consider keepingpets outside of the room if they interfere with sleep \\
- No heavy meals within three hours of bedtime & \\
- Keep a relaxing routine (warm bath, wind down) & \\
- Avear comfortable clothing & \\
- Do not use the bed for watching TV and reading & \\
\hline
\end{tabular}

\section{Pharmacological Treatment}

Medications may be used early in the treatment for relief of temporary/ situational insomnia when an immediate response is required. More often it is reserved for patients demonstrating significant consequences of insomnia refractory to other treatments. As with all medications prescribed to the elderly, lowest doses and shortest duration of administration are preferred. Medication use should be tapered gradually to prevent rebound or withdrawal symptoms when treatment is completed. If higher doses are used it is important to monitor for dependency and abuse especially in those who are resistant to tapering and those requesting for higher doses of the medications. ${ }^{17,18,19}$

\section{A. Benzodiazepines}

Benzodiazepine decreases sleep latency and nocturnal awakenings. However, benzodiazepines such as lorazepam, diazepam etc. may increase the risk of memory impairment, falls, fracture, vehicular accidents, increase emergency department visits and unnecessary hospitalisations. Long term usage can lead to psychological dependence and there is an increased risk of addiction and abuse. Tolerance can also develop over time requiring higher dosages to be effective. Therefore, whenever possible, its use should be minimised in the elderly.

\section{B. N on-benzodiazepine Hypnotics}

This class of drugs is commonly known as the z-drugs (zolpidem, zopiclone) with similar actions to benzodiazepines. It reduces sleep latency. In the elderly it should be used for short term (not more than three months) as it causes confusion and increases the risk of falls and fractures in adults with dementia and cognitive impairment. There is also potential for abuse, in higher dosages the medication can cause stimulation, euphoria and anxiolysis in some patients. Uncommon complex sleep related behaviours (sleep driving, sleep eating) have been reported in patients taking higher dosages. 


\section{Melatonin and Melatonin receptor agonist}

Melatonin receptor agonists (Ramelteon) and melatonin reduce sleep latency and increase sleep duration. Their side effects are usually mild such as gastrointestinal disturbances and neurological such as giddiness and headache. They are relatively safe and there are no documented rebound insomnia or withdrawal side effects. Melatonin is a health supplement that is not regulated by the Health Science Authority and is available as an over the counter product in Singapore. Melatonin receptor agonists are not approved for sale in Singapore and hence not available. Circadin (Melatonin prolong release tablets) is available in Singapore for the short-term treatment of primary insomnia characterised by poor quality of sleep in the older patients.

\section{O ver the counter sleep aid and antihistamines}

These medications decrease sleep latency but they produce rapid tolerance and are highly anticholinergic. The side effects include dry mouth, constipation, blurring of visions and acute retention of urine. They should be avoided in the elderly as it increases the risk of cognitive decline and impairment.

\section{E. Antidepressants}

Many antidepressants (Mirtazapine, fluvoxamine, amitriptyline and nortriptyline) have sedative side effects and are sometimes used to treat insomnia especially in those with concurrent depressions. However, most are also highly anticholinergic and increases the risk of falls and accidents in the elderly. The only drug that has been approved for the treatment of insomnia by the Food and Drug Administration (FDA) is tricyclic antidepressant Doxepin. Its mechanism is unknown but compared to placebo, doxepin at $3 \mathrm{mg}$ to $6 \mathrm{mg}$ improves sleep efficiency and latency. ${ }^{20}$ The side effect profile at these dosages is favourable in the elderly. In Singapore it is available as an inexpensive $10 \mathrm{mg}$ dosage.

It must be emphasised that the management of insomnia in elderly is behavioural therapy as first line and combination with medications if the intervention is ineffective. All medication increases the risk of falls and the use of over the counter sleeping aid should be avoided in the elderly. It is recommended that medications should be avoided or be used only for the shortest period of time in the elderly. Currently, the medications considered the safest and most effective prescription sleeping aids for geriatric patients are zolpidem, zopiclone, melatonin and agonist, Ramelteon (not available in Singapore) and low dose tricyclic antidepressant, Doxepin. For sleep-onset insomnia, one can consider prescribing a shorter acting agent such as zolpidem. For sleep maintenance insomnia, a longer acting agent such as zolpidem CR, zopiclone and doxepin, can be used. In patients with concomitant anxiety, consider a selective serotonin reuptake inhibitors (SSRI).

Herbal therapy or folk remedies usage is not uncommon as a form of treatment in traditional Singapore. Common herbal therapy includes St John's wort, Chamomile, kava,etc. Most are untested and has no known efficacy or safety profile and may even be toxic and generally, advice should be given to avoid them.

\section{CONCLUSION}

In the elderly, insomnia can affect personal well-being and lead to significant morbidity. Many elderly experience difficulties with falling asleep, or waking up too early, which leads to daytime impairment. Sleep assessment should be considered as an integral part of a comprehensive geriatric assessment. Identification and treatment of the many potentially contributing factors are essential to effective therapy. For most patients, behavioural therapy should be the initial treatment. Avoid benzodiazepines and other sedative hypnotics as first choice for treatment. If medications are needed, they should always be used in combination with behavioural therapy and at the lowest effective dose.

\section{REFEREN CES}

1. Tan NC, Tan MS, Hwang SW, Teo CC, Lee ZK, Soh JY, Koh YL, How CH. Sleep time and pattern of adult individuals in primary care in an Asian urbanized community: A crosssectional study. Medicine. 2016 Aug;95(35).

2. Chiu HF, Leung T, Lam LC, Wing YK, Chung DW, Li SW, Chi I, Law WT, Boey KW. Sleep problems in Chinese elderly in Hong Kong. Sleep. 1999 Sep 1;22(6):717-26.

3. Ohayon MM. Epidemiology of insomnia: what we know and what we still need to learn. Sleep medicine reviews. 2002 May 1;6(2):97-111.

4. Masters PA. Insomnia. Annals of internal medicine. 2014 Oct 7; 161(7):ITC1-.

5. Alvaro PK, Roberts RM, Harris JK. A systematic review assessing bidirectionality between sleep disturbances, anxiety, and depression. Sleep. 2013 Jul 1;36(7):1059-68.

6. Krystal AD. Psychiatric comorbidity: the case for treating insomnia. Sleep Medicine Clinics. 2006 Sep 1;1(3):359-65.

7. Agency for Healthcare Research and Quality. Management of Insomnia Disorder in Adults: Current State of the Evidence. Available at:https://effectivehealthcare.ahrq.gov/ topics/insomnia/clinician . [Assessed 20 March 2019]

8. National Guideline Clearing house [Internet]. Rockville (MD): Agency for Healthcare Research and Quality (AHRQ); [1997] -. Guideline summary: Clinical guideline for the treatment of primary insomnia in middle-aged and older adults. May, 2014. [Assessed 20 March 2019]. Available from: https://www.multiplechronicconditions.org/assets/ pdf/Insomnia\%20Guidelines/AHRQ_2014_Clinical_guideline_for_the_treatment_of_ primary_insomnia_in_middle-aged_and_older_adults.pdf

9. Ringdahl EN, Pereira SL, Delzell JE. Treatment of primary insomnia. The Journal of the American Board of Family Practice. 2004 May 1;17(3):212-9.

10. Maness DL, Khan M. Nonpharmacologic Management of Chronic Insomnia. American family physician. 2015 Dec 15;92(12).

11. Ong JC, Manber R, Segal Z, Xia Y, Shapiro S, Wyatt JK. A randomized controlled trial of mindfulness meditation for chronic insomnia. Sleep. 2014 Sep 1;37(9):1553-63.

12. Zhang JX, Liu XH, Xie XH, Zhao D, Shan MS, Zhang XL, Kong XM, Cui H. Mindfulness-based stress reduction for chronic insomnia in adults older than 75 years: a randomized, controlled, single-blind clinical trial. Explore. 2015 May 1;11(3):180-5.

13. King AC, Oman RF, Brassington GS, Bliwise DL, Haskell WL. Moderate-intensity exercise and self-rated quality of sleep in older adults: a randomized controlled trial. Jama. 1997 Jan 1;277(1):32-7.

14. Sloane PD, Figueiro M, Cohen L. Light as therapy for sleep disorders and depression in older adults. Clinical geriatrics. 2008 Mar 1;16(3):25.

15. Lack L, Wright H, Kemp K, Gibbon S. The treatment of early-morning awakening insomnia with 2 evenings of bright light. Sleep. 2005 May 1;28(5):616-23.

16. Kwok T, Leung PC, Wing YK, Ip I, Wong B, Ho DW, Wong WM, Ho F. The effectiveness of acupuncture on the sleep quality of elderly with dementia: a within-subjects trial. Clinical interventions in aging. 2013; 8:923.

17. Brewster GS, Riegel B, Gehrman PR. Insomnia in the Older Adult. Sleep medicine clinics. 2018 Mar 1;13(1):13-9.

18. Roszkowska J, Geraci SA. Management of insomnia in the geriatric patient. The American journal of medicine. 2010 Dec 1;123(12):1087-90. 
19. Matheson E, Hainer BL. Insomnia: Pharmacologic Therapy. American family physician. 2017 Jul;96(1):29-35.

20. Roth T, Rogowski R, Hull S, Schwartz H, Koshorek G, Corser B, Seiden D, Lankford A. Efficacy and safety of doxepin $1 \mathrm{mg}, 3 \mathrm{mg}$, and $6 \mathrm{mg}$ in adults with primary insomnia. Sleep. 2007 Nov 1;30(11):1555-61.

\section{LEARN IN G POINTS}

- Incidence of insomnia increases with age. It can include difficulty falling asleep at the start of the sleep period, waking up and difficulty falling back asleep, and waking up early and unable to get back to sleep. Difficulty staying asleep and early morning awakening are common in the elderly. W aking up early can also be due to advanced sleep phase syndrome.

- A thorough history (both medical and psychiatric) and physical examination are required when diagnosing insomnia.

- Behavioural therapy should be the first line of therapy and sleep hygiene education can be conducted by the family physician in the clinic. If there is little improvement, referral should be considered to a specialist geriatrician or psychiatrist for Cognitive Behavioural Therapy for Insomnia (CBT-I) or multicomponent therapy.

- C BT - I has been shown to be more effective than medictions for long term management of insomnia in the older adults.

- Medications should be used sparingly and when possible be discontinued. If medications are needed, it should be used in conjunction with behavioural interventions.

- W herever possible, it is wise to avoid using benzodiazepines or other sedative hypnotics in older adults as a first choice for insomnia, agitation, or delirium.

- Currently, the appropriate drugs to use in the elderly are zolpidem, melatonin, zopiclone and doxepin. 